\title{
Transition metal-free hydration of nitriles to amides mediated by $\mathrm{NaOH}$
}

\author{
N Naresh Kumar Reddy ${ }^{1}$, Sadu Nageswara Rao ${ }^{1}$, Rajendra D Patil ${ }^{2}$ and Subbarayappa Adimurthy ${ }^{1 *}$ \\ ${ }^{1}$ Central Salt \& Marine Chemicals Research Institute, Council of Scientific \& Industrial Research, Gujarat, India \\ ${ }^{2}$ Department of Chemistry, School of Science, Sandip University, Maharashtra, India
}

\begin{abstract}
A transition metal-free $\mathrm{NaOH}$ mediated hydration of organo nitriles to amides under mild reaction conditions has been described. Both aliphatic and aromatic/hetero nitriles were smoothly converted into corresponding amides in moderate to good yields.
\end{abstract}

\section{Introduction}

Amides are an important key intermediate in many organic transformations, as well as they are basic building blocks in biological molecules, agrochemicals, polymers etc. ${ }^{[1]}$ The amide linkage is one of the most important functional group in nature, because they are key connecter in peptides and proteins in living organisms. ${ }^{[2]}$ The synthesis of amides were also significant importance in the field of pharmaceutical, medicinal chemistry. Amides are present in around $25 \%$ of top-selling in pharmaceuticals industry. Compared to secondary and tertiary amides, the primary amides are important intermediates in organic synthesis and these are raw materials for the synthesis of plastics, detergents and lubricants. ${ }^{[3]}$ Due to the numerous applications of amides, a number of elegant methods have been developed in recent years. Generally, the amide bonds are formed by the condensation of carboxylic acid and esters with amines, ${ }^{[4]}$ or coupling reactions between alcohol/aldehydes with amines ${ }^{[5]}$ and hydroamination of unsaturated hydrocarbons. ${ }^{[6]}$ Apart from these methods, hydration of nitrilesis one of the classic transformation as well as simple and straightforward method for the synthesis of variety of amides, in green chemistry point of view, the nitrile hydration methods were also promote as atom efficiency and avoids the generation of environmental waste. Based on these advantages, the hydration of nitriles to amides is a wellestablished method in the pharmaceutical industry for the synthesis of various amides in large scale production. Recently various groups were reported the hydration of the nitriles to amides using different catalysts such as acids, bases, ionic liquids, and transition metals. ${ }^{[7,8]}$

Recently, some green protocols such as, microwave assisted reactions, TBAH catalyst, super basic system $\mathrm{DMSO}-\mathrm{CsOH}, \mathrm{KO}^{t} \mathrm{Bu}$ have been recently reported (Scheme 1). ${ }^{[9]}$ In many instances the practicality of the methods have limitations such as harsh reaction conditions, high temperature, strong bases, requirement of precious metal combinations. Therefore, the development of convenient, practical methods for the hydration of nitriles to amides under transition metal-free conditions still holds its relevance.

\section{Results and Discussion}

In continuation of our interest on the development of green and sustainable methods for amides, ${ }^{[10]}$ we describe herein hydration

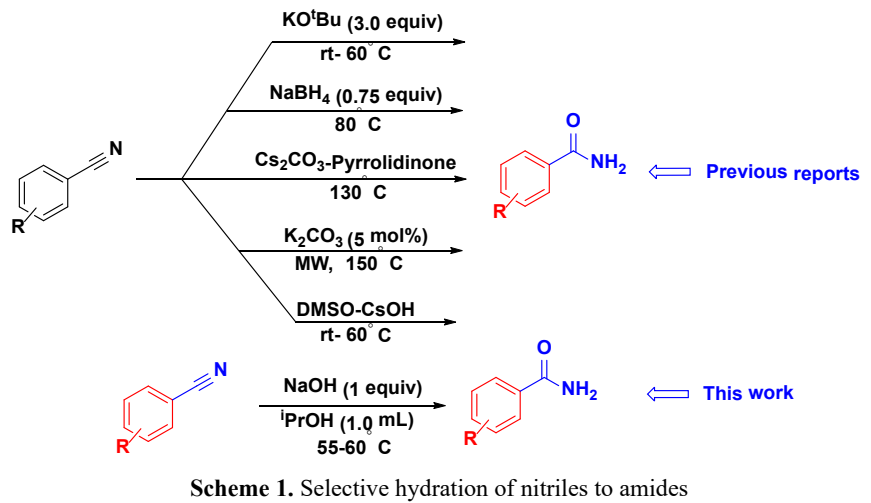

of nitriles to the corresponding amides using inexpensive and commercially available $\mathrm{NaOH}$ as promoter under metal-free mild conditions.We initiated our studies with benzonitrile (1a) as a model substrate, using $\mathrm{NaOH}$ as a promote at room temperature with isopropyl alcohol (IPA) as solvent, under these conditions the desired product (2a) was obtained in $21 \%$ isolated yield after $24 \mathrm{~h}$ (Table 1, entry 1).

Based on the initial observation, we raised the reaction temperature (from room temperature) to 55 and $60{ }^{\circ} \mathrm{C}$ under the same reaction conditions; the yield of the desired product was significantly increased to $86 \%$ and $93 \%$ respectively (Table 1 , entries $2 \& 3$ ). When the amount of base was reduced to 0.5 equivalents, the yield was dropped to $65 \%$ (Table 1, entry 4). Under the same conditions, we tested the reaction with other inorganic bases like, $\mathrm{K}_{2} \mathrm{CO}_{3}$, and $\mathrm{Na}_{2} \mathrm{CO}_{3}$ no product formation was observed (Table 1 , entries 5 \& 6). However, lower yield of the product was obtained with $\mathrm{LiOH} . \mathrm{H}_{2} \mathrm{O}$ and $\mathrm{CsOH}$ as base (Table 1 , entries7 \& 8). With $\mathrm{KOH}$ as a promoter, $85 \%$ yield of 2 a was obtained

${ }^{\star}$ Correspondence to: Subbarayappa Adimurthy, Central Salt \& Marine Chemicals Research Institute, Council of Scientific \& Industrial Research, G.B. Marg, Bhavnagar-364 002, Gujarat, India, Fax: +91-278-2567562; E-mail: adimurthy@csmcri.res.in

Key words: nitriles, base, hydration, amides

Received: March 26, 2018; Accepted: April 16, 2018; Published: April 19, 2018 
Table 1. Optimization of reaction conditions $\mathrm{s}^{\mathrm{a}}$

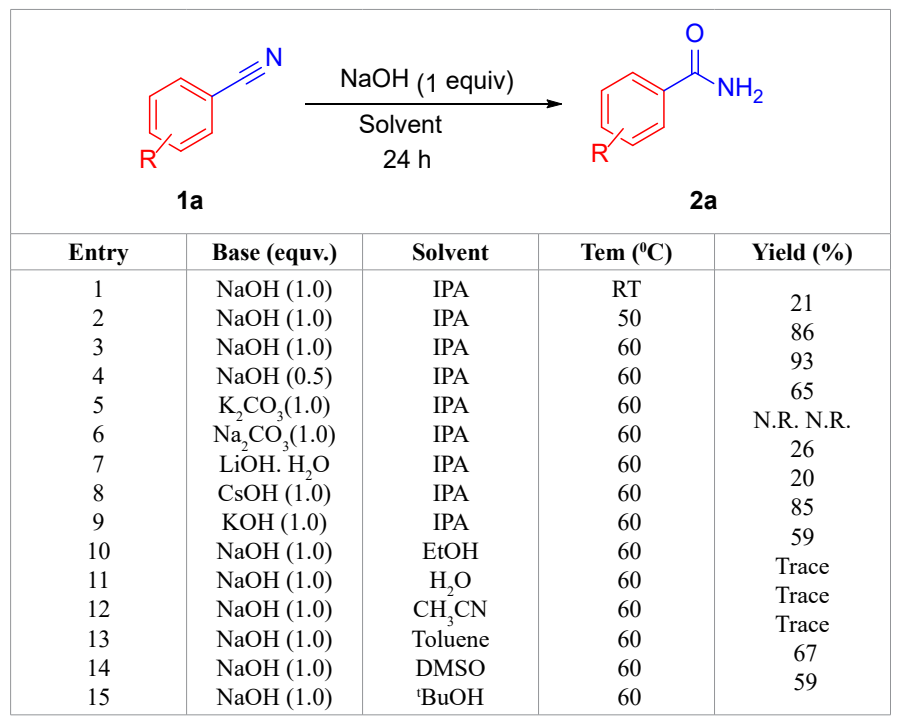

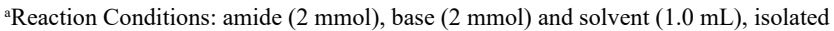
yields.

under the same conditions (Table 1, entry 9). Further, keeping with $\mathrm{NaOH}$ as promoter, different solvents $\left(\mathrm{EtOH}, \mathrm{H}_{2} \mathrm{O}, \mathrm{CH}_{3} \mathrm{CN}\right.$, toluene, DMSO and ${ }^{t} \mathrm{BuOH}$ ) were screened to examine the yield of the product, lower yield to no reaction was observed with these solvents (Table 1, entries 10-15). After screening for various parameters, the optimum conditions identified for the present transformation are asfollows: 1.0 equiv. of $\mathrm{NaOH}$ as base, and $1.0 \mathrm{~mL}$ of IPA at $60^{\circ} \mathrm{C}, 24 \mathrm{~h}$ reaction time (Table 1, entry 3 ).

With the optimized conditions in hand, the substrate scope of the reaction for the hydration of various nitriles were investigated (Table 2).The reactions were found to be very facile with both electron rich and electron deficient groups. In electron donating groups such as $-\mathrm{Me},-\mathrm{OMe},-\mathrm{Ph}$ at para position of benzonitriles (2a-2d) to gives corresponding amides are excellent yields. Where as in the case of halogen assisted benzonitriles at the para position such as $-\mathrm{F},-\mathrm{Cl},-\mathrm{Br}$, and $-\mathrm{I}$ to affords the desired products $\mathbf{2 e - 2 h}$ were obtained in good to excellent yields (69-97\%). The p-tolyl benzonitrile also underwent to the same conditions and provided the desired amide product $2 \mathbf{i}$ in $71 \%$ yield. Further, in the presence of meta substituted benzonitrles like-OMe, - $\mathrm{Me}(\mathbf{2} \mathbf{j}-\mathbf{2 k})$ in this case also reaction underwent smoothly and affords to excellent yields. For halogen substituted either at meta or ortho position of benzonitriles gave the corresponding amides $\mathbf{2 l}-\mathbf{p}$ in good to moderate yields (61-73\%). And 2-methoxy benzonitrile subjected under standard conditions to affords corresponding amide $\mathbf{2 q}$ is $89 \%$. The hydration of $\alpha$-cyano naphthalene under the present conditions afford the naphthylamide $2 \mathbf{r}$ in $69 \%$ yield. To validate the present protocol, the product 2a was obtained at gram scale in $92 \%$ yield using $10.0 \mathrm{mmol}$ (1.03 g) of $\mathbf{1 a}$.

We then evaluated the hydration of heteroaromatic and aliphatic nitriles under the above optimized conditions (Table 3). Particularly, the heteroaromatic amides are the key intermediates in the preparation of 2-pyridyl urea derivatives which are potent inhibitors of gastric acid secretion. ${ }^{[11]}$ Such amides have been prepared through oxidative coupling of corresponding aldehydes and terminal alkynes using $\mathrm{Cu}(\mathrm{OTf})_{2} /$ $\mathrm{I}_{2}$ system. ${ }^{[12]}$ Notably, heteroaromatic amides such as thiophene- carboxamides $\mathbf{3} \mathbf{a}-\mathbf{b}$, picolinamides $\mathbf{3 c}$-e, pyrazine-carboxamide $\mathbf{3 f}$ and 4-(imidazo[1,2-a]pyridin-2-yl)benzamide $3 \mathbf{g}$, could be obtained in good yields (51-89\%) under the present conditions. To our delight the optimized conditions were also applicable to aliphatic nitriles and afford the corresponding aliphatic amides $\mathbf{3 h}-\mathbf{i}$ in good yields (59-63\%).

Based on experimental observations and literature reports ${ }^{[\mathrm{dd}, 9 \mathrm{~d}]}$ an ionic mechanism has been proposed for the present transformation (Scheme 2). Initially, IPA in presence of $\mathrm{NaOH}$ forms iso-propoxy anion intermediate, it reacts with nitrile and produces theintermediate $\mathbf{I}$ which exist as keto - enol form and easily hydrolyzes to form corresponding desired amide.

Table 2. Substrates scope of the benzonitriles
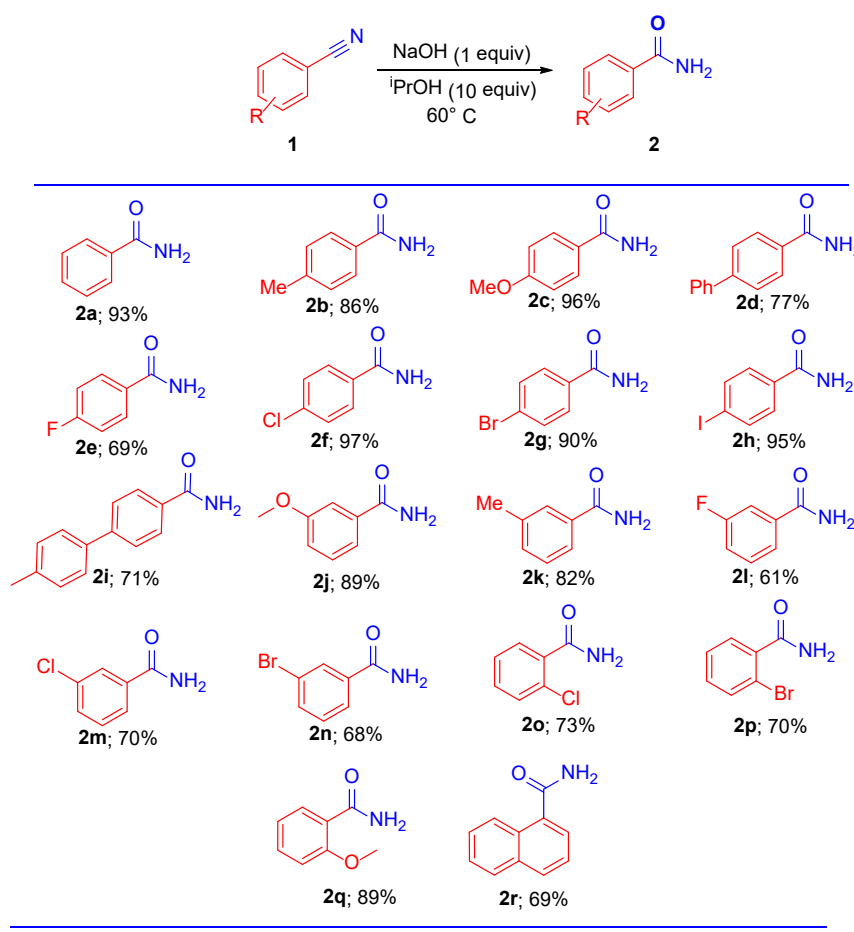

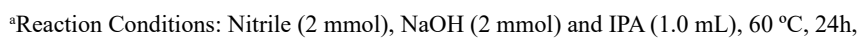
isolated yields.

Table 3. Substrates scope of the hetero and aliphatic nitriles ${ }^{a}$

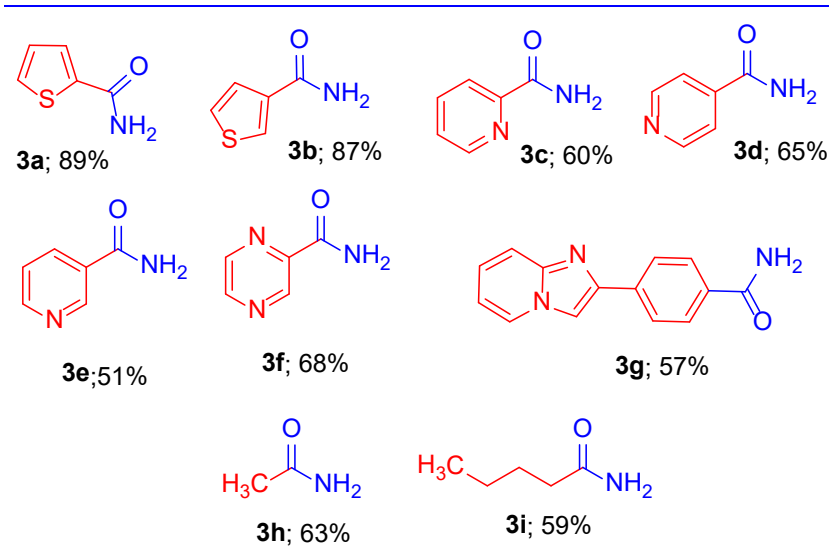

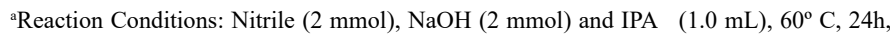
isolated yields 
In conclusion, we have developed an efficient protocol for the hydration of various benzonitriles to corresponding benzamides using inexpensive and commercially available base $(\mathrm{NaOH})$ under very mild conditions $\left(60^{\circ} \mathrm{C}\right)$. The conditions also applicable to heteroaromatic nitriles and aliphatic nitriles and afford the good to excellent yields. The present method represents a significant development for the hydration of nitriles under transition metalfree conditions.

\section{Experimental Section}

\section{General information}

All commercially available chemicals and reagents were used without anyfurther purification unless otherwise indicated. ${ }^{1} \mathrm{H}$ and ${ }^{13} \mathrm{C}$ NMR spectra were recorded at 500, 600 and 125, $150 \mathrm{MHz}$,respectively. The spectra were recorded in $\mathrm{CDCl}_{3}$ andDMSO- $\mathrm{d}_{6}$ as a solvent. Multiplicity was indicated asfollows: s (singlet); d (doublet); $\mathrm{t}$ (triplet); $\mathrm{m}$ (multiplet); dd (doublet of doublets), etc. Couplingconstants $(J)$ were given in $\mathrm{Hz}$. Chemical shifts are reported in $\delta$ relative to TMS as an internalstandard. The peaks around $\delta$ values of $7.26\left({ }^{1} \mathrm{H}\right.$ NMR), $77.0\left({ }^{13} \mathrm{C} \mathrm{NMR}\right)$ correspond to $\mathrm{CDCl}_{3}$. The peaks around $\delta$ values of 2.50 ( ${ }^{1} \mathrm{H}$ NMR), $39.9\left({ }^{13} \mathrm{C} \mathrm{NMR}\right)$ are corresponding to DMSO. The peak around $\delta$ values of 3.35 ( ${ }^{1} \mathrm{H}$ NMR) is corresponding to the $\mathrm{H}_{2} \mathrm{O}$ present in DMSO solvent. Progress of the reactions was monitored by thin layer chromatography (TLC). Silica gel 100-200mesh size was used for column chromatography using a hexane/ethyl acetate eluent unless otherwise indicated.

\section{General experimental procedure for the synthesis of benzamide from benzonitrile (3a)}

A $20 \mathrm{~mL}$ round bottomed flask was charged with benzonitrile (2 $\mathrm{mmol})$, sodium hydroxide ( $2 \mathrm{mmol}$ ) dissolved in isopropyl alcohol (1.0 $\mathrm{mL}$ ). Then the reaction mixture was placed at indicated temperature and time, and the progress of the reaction was monitored by TLC. After completion of the reaction, the crude mixture was dissolved with dichloromethane and filtered the mixture and evaporated to dryness. The residue was then purified by column chromatography (hexane/ EtOAc) to get the pure product. All amide products were characterized by NMR.

\section{Characterization data}

Benzamide (2a)<smiles>NC(=O)c1ccccc1</smiles>

(Eluent: 40\% EtOAc/hexane); 93\% yield (225.1 mg); white solid; ${ }^{1} \mathrm{H}$ NMR $\left(500 \mathrm{MHz}, \mathrm{CDCl}_{3}\right): \delta 7.82(\mathrm{~s}, 1 \mathrm{H}), 7.81(\mathrm{~d}, J=2.0 \mathrm{~Hz}, 1 \mathrm{H}), 7.53$ $(\mathrm{t}, J=6.5 \mathrm{~Hz}, 1 \mathrm{H}), 7.44(\mathrm{t}, J=7.0 \mathrm{~Hz}, 2 \mathrm{H}), 6.17(\mathrm{br}, \mathrm{NH}, 2 \mathrm{H}) \cdot{ }^{13} \mathrm{C} \mathrm{NMR}$ $\left(125 \mathrm{MHz}, \mathrm{CDCl}_{3}\right) \delta 169.5,133.3,131.9,128.5,127.3$.

4-Methylbenzamide (2b)<smiles>Cc1ccc(C(N)=O)cc1</smiles>

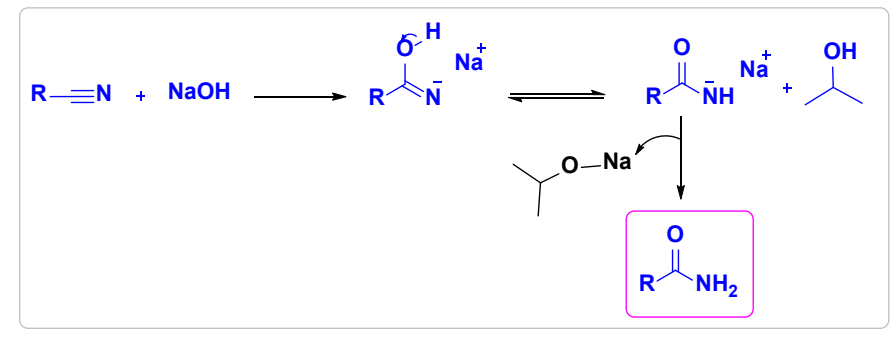

Scheme 2. A proposed mechanism for hydration of nitriles

(Eluent: $40 \%$ EtOAc/hexane); $86 \%$ yield $(232.2 \mathrm{mg})$; white solid; ${ }^{1} \mathrm{H}$ NMR $\left(500 \mathrm{MHz}, \mathrm{CDCl}_{3}\right): \delta 7.72(\mathrm{~d}, J=8.0 \mathrm{~Hz}, 2 \mathrm{H}), 7.25(\mathrm{~d}, J=8.0 \mathrm{~Hz}$, $2 \mathrm{H}), 6.10$ (br, NH, 2H). ${ }^{13} \mathrm{C} \mathrm{NMR}\left(125 \mathrm{MHz}, \mathrm{CDCl}_{3}\right) \delta 169.5,142.5$, $130.5,129.2,127.3,21.4$.

4-Methoxybenzamide (2c)<smiles>COc1ccc(C(N)=O)cc1</smiles>

(Eluent: 30\% EtOAc/hexane); 92\% yield (278 mg); white solid; ${ }^{1} \mathrm{H}$ $\operatorname{NMR}\left(600 \mathrm{MHz}, \mathrm{CDCl}_{3}\right): \delta 7.79(\mathrm{~d}, J=7.0 \mathrm{~Hz}, 2 \mathrm{H}), 6.94(\mathrm{~d}, J=7.5 \mathrm{~Hz}$, 2H), 5.99 (br, NH, 2H), $3.86(\mathrm{~S}, 3 \mathrm{H}) .{ }^{13} \mathrm{C} \mathrm{NMR}\left(150 \mathrm{MHz}, \mathrm{DMSO}-\mathrm{d}_{6}\right) \delta$ $167.9,162.0,129.8,127.0,113.8,55.8$.

3-methoxybenzamide (2d)<smiles>COc1cccc(C(N)=O)c1</smiles>

(Eluent: 35\% EtOAc/hexane); 89\% yield (270.1 mg); white solid; ${ }^{1} \mathrm{H}$ $\operatorname{NMR}\left(600 \mathrm{MHz}, \mathrm{CDCl}_{3}\right): \delta 7.40(\mathrm{~s}, 1 \mathrm{H}), 7.35(\mathrm{~m}, 2 \mathrm{H}), 7.08$ (d, $J=7.5$ $\mathrm{Hz}, 1 \mathrm{H}), 6.10$ (br, NH, 2H), 3.85 (s, 3H). ${ }^{13} \mathrm{C} \mathrm{NMR}\left(150 \mathrm{MHz}, \mathrm{CDCl}_{3}\right) \delta$ $169.2,159.9,134.8,129.7,119.2,118.3,112.6,55.5$.

3-Methylbenzamide (2e)<smiles>Cc1cccc(C(N)=O)c1</smiles>

(Eluent: 40\% EtOAc/hexane); 82\% yield (221.5 mg); white solid; ${ }^{1} \mathrm{H}$ NMR (600 MHz, $\left.\mathrm{CDCl}_{3}\right): \delta 7.65(\mathrm{~s}, 1 \mathrm{H}), 7.59(\mathrm{~d}, J=5.5 \mathrm{~Hz}, 1 \mathrm{H}), 7.30$ $(\mathrm{t}, J=6.0 \mathrm{~Hz}, 2 \mathrm{H}), 6.49$ (br, NH, 2H), 2.37 (s, 3H). ${ }^{13} \mathrm{C} \mathrm{NMR}(150 \mathrm{MHz}$, $\left.\mathrm{CDCl}_{3}\right) \delta 170.1,138.4,133.4,132.6,128.4,128.1,124.3,21.3$.

2-methoxybenzamide (2f)<smiles>COc1ccccc1C(N)=O</smiles> 
(Eluent: 30\% EtOAc/hexane); 84\% yield (255.1 mg); white solid; ${ }^{1} \mathrm{H}$ $\operatorname{NMR}\left(600 \mathrm{MHz}, \mathrm{CDCl}_{3}\right): \delta 7.22(\mathrm{dd}, J=5.0 \mathrm{~Hz}, 1 \mathrm{H}), 7.49(\mathrm{~m}, 1 \mathrm{H}), 7.08$ $(\mathrm{t}, J=6.0 \mathrm{~Hz}, 1 \mathrm{H}), 7.00(\mathrm{~d}, J=6.5 \mathrm{~Hz}, 1 \mathrm{H}), 6.06(\mathrm{br}, \mathrm{NH}, 2 \mathrm{H}), 3.97(\mathrm{~s}$, $3 \mathrm{H}) .{ }^{13} \mathrm{C}$ NMR $\left(150 \mathrm{MHz}, \mathrm{CDCl}_{3}\right) \delta 167.1,157.8,133.4,132.6,121.3$, $120.8,111.4,56.0$.

4'-Methyl-[1, 1'-biphenyl]-4-carboxamide (2g)

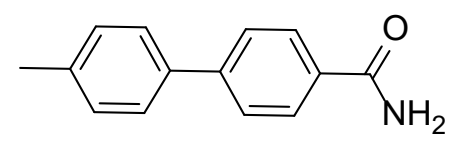

(Eluent: $40 \%$ EtOAc/hexane); $71 \%$ yield $(301.6 \mathrm{mg})$; white solid; ${ }^{1} \mathrm{H}$ $\operatorname{NMR}\left(600 \mathrm{MHz}, \mathrm{CDCl}_{3}\right): \delta 7.86(\mathrm{~d}, J=7.0 \mathrm{~Hz}, 2 \mathrm{H}), 7.64(\mathrm{~d}, J=7.0 \mathrm{~Hz}$, $2 \mathrm{H}), 7.50(\mathrm{~d}, J=6.5 \mathrm{~Hz}, 2 \mathrm{H}), 7.26(\mathrm{~d}, J=6.5 \mathrm{~Hz}, 2 \mathrm{H}), 6.08(\mathrm{br}, \mathrm{NH}$, 2H), 2.39 (s, 3H). ${ }^{13} \mathrm{C}$ NMR (150 MHz, DMSO-d $)$ ) $\delta$ 168.0, 143.1, 137.9, $136.8,133.3,130.1,128.6,127.2,126.6,21.2$.

4-Fluorobenzamide (2h)<smiles>NC(=O)c1ccc(F)cc1</smiles>

(Eluent: 40\% EtOAc/hexane); 69\% yield (193.1 mg); white solid; ${ }^{1} \mathrm{H}$ NMR $\left(500 \mathrm{MHz}, \mathrm{CDCl}_{3}\right): \delta 7.83(\mathrm{~m}, 2 \mathrm{H}), 7.12(\mathrm{t}, J=9.0 \mathrm{~Hz}, 2 \mathrm{H}), 6.06$ (br, NH, 2H). ${ }^{13} \mathrm{C}$ NMR $\left(125 \mathrm{MHz}, \mathrm{CDCl}_{3}\right) \delta 168.3,166.0,(\mathrm{~d}, J=251.2$ $\mathrm{Hz}), 164.0,129.8$ (d, $J=8.75 \mathrm{~Hz}), 129.7,115.8$ (d, $J=0.17 \mathrm{~Hz}), 115.6$.

3-Fluorobenzamide (2i)<smiles>NC(=O)c1cccc(F)c1</smiles>

(Eluent: $40 \%$ EtOAc/hexane); $61 \%$ yield $(170.7 \mathrm{mg})$; white solid; ${ }^{1} \mathrm{H}$ NMR $\left(600 \mathrm{MHz}, \mathrm{CDCl}_{3}\right): \delta 7.56(\mathrm{t}, J=6.5 \mathrm{~Hz}, 1 \mathrm{H}), 7.43(\mathrm{q}, J=$ $6.5 \mathrm{~Hz}, 1 \mathrm{H}), 7.23(\mathrm{t}, \mathrm{J}=7.0 \mathrm{~Hz}, 1 \mathrm{H}), 6.12(\mathrm{br}, \mathrm{NH}, 2 \mathrm{H}) .{ }^{13} \mathrm{C}$ NMR $(150$ $\left.\mathrm{MHz}, \mathrm{CDCl}_{3}\right) \delta 168.1,163.6(\mathrm{~d}, J=246 \mathrm{~Hz}), 162.0,135.7,130.4(\mathrm{~d}, J=$ $7.5 \mathrm{~Hz}), 130.3,122.89$ (d, $J=3.0 \mathrm{~Hz}), 122.87,119.2,119.0,114.9,114.7$.

4-Bromobenzamide (2j)<smiles>NC(=O)c1ccc(Br)cc1</smiles>

(Eluent: 40\% EtOAc/hexane); 90\% yield (358.0 mg); white solid; ${ }^{1} \mathrm{H}$ NMR $\left(600 \mathrm{MHz}, \mathrm{CDCl}_{3}\right): \delta 7.68(\mathrm{~d}, J=7.0 \mathrm{~Hz}, 2 \mathrm{H}), 7.60(\mathrm{~d}, J=7.5 \mathrm{~Hz}$, 2H), 6.05 (br, NH, 2H). ${ }^{13} \mathrm{C}$ NMR (150 MHz, $\mathrm{CDCl}_{3}$ ) $\delta 168.2,132.1$, 131.9, 128.9, 126.8 .

3-Bromobenzamide (2k)<smiles>NC(=O)c1cccc(Br)c1</smiles>

(Eluent: 35\% EtOAc/hexane); 68\% yield (271.3 mg); white solid; ${ }^{1} \mathrm{H}$ NMR $\left(600 \mathrm{MHz}, \mathrm{CDCl}_{3}\right): \delta 7.97(\mathrm{~s}, 1 \mathrm{H}), 7.72(\mathrm{~d}, J=6.5 \mathrm{~Hz}, 1 \mathrm{H}), 7.67$ $(\mathrm{d}, J=7.0 \mathrm{~Hz}, 1 \mathrm{H}), 7.33(\mathrm{t}, J=6.0 \mathrm{~Hz}, 1 \mathrm{H}), 6.11(\mathrm{br}, \mathrm{NH}, 2 \mathrm{H}) \cdot{ }^{13} \mathrm{C}$ NMR $\left(150 \mathrm{MHz}, \mathrm{CDCl}_{3}\right) \delta 167.8,135.2,135.0,130.6,130.2,125.8,122.8$.

2-Bromobenzamide (2l)<smiles>CC(=O)c1ccc(Cl)cc1</smiles>

(Eluent: $30 \%$ EtOAc/hexane); $70 \%$ yield $(278.3 \mathrm{mg})$; white solid; ${ }^{1} \mathrm{H}$ $\operatorname{NMR}\left(600 \mathrm{MHz}, \mathrm{CDCl}_{3}\right): \delta 7.64(\mathrm{~m}, 1 \mathrm{H}), 7.62(\mathrm{~d}, J=6.5 \mathrm{~Hz}, 1 \mathrm{H}), 7.38$ $(\mathrm{t}, J=6.0 \mathrm{~Hz}, 1 \mathrm{H}), 7.30(\mathrm{~m}, 1 \mathrm{H}), 6.11(\mathrm{br}, \mathrm{NH}, 2 \mathrm{H}) .{ }^{13} \mathrm{C} \mathrm{NMR}(150 \mathrm{MHz}$, $\left.\mathrm{CDCl}_{3}\right) \delta 169.1,136.5,131.7,129.9,127.6,119.2$.

4-Chlorobenzamide (2m)<smiles>NC(=O)c1ccc(Cl)cc1</smiles>

(Eluent: $40 \%$ EtOAc/hexane); 97\% yield (300.2 mg); white solid; ${ }^{1} \mathrm{H}$ NMR $\left(600 \mathrm{MHz}, \mathrm{CDCl}_{3}\right): \delta 7.75(\mathrm{~d}, J=7.0 \mathrm{~Hz}, 2 \mathrm{H}), 7.41(\mathrm{t}, J=6.5 \mathrm{~Hz}$, $2 \mathrm{H}), 5.82$ (br, $\mathrm{NH}, 2 \mathrm{H}) \cdot{ }^{13} \mathrm{C} \mathrm{NMR}\left(150 \mathrm{MHz}, \mathrm{CDCl}_{3}\right) \delta 168.2$, 138.4, 131.6, 129.0, 128.8 .

2-Chlorobenzamide (2n)<smiles>NC(=O)c1ccccc1Cl</smiles>

(Eluent: $30 \%$ EtOAc/hexane); $73 \%$ yield $(225.8 \mathrm{mg})$; white solid; ${ }^{1} \mathrm{H}$ NMR $\left(600 \mathrm{MHz}, \mathrm{CDCl}_{3}\right): \delta 7.79(\mathrm{~d}, J=7.0 \mathrm{~Hz}, 1 \mathrm{H}), 7.42(\mathrm{~d}, J=6.5$ $\mathrm{Hz}, 1 \mathrm{H}), 7.39$ (d, $J=5.5 \mathrm{~Hz}, 1 \mathrm{H}), 7.35(\mathrm{t}, J=7.0 \mathrm{~Hz}, 1 \mathrm{H}), 6.37$ (br, NH, $2 \mathrm{H}) .{ }^{13} \mathrm{C} \mathrm{NMR}\left(150 \mathrm{MHz}, \mathrm{CDCl}_{3}\right) \delta 168.0,133.7,131.9,130.88,130.81$, 130.4, 127.2.

3-Chlorobenzamide (2o)<smiles>NC(=O)c1cccc(Cl)c1</smiles>

(Eluent: 40\% EtOAc/hexane); $70 \%$ yield $(218.2 \mathrm{mg})$; white solid; ${ }^{1} \mathrm{H}$ NMR $\left(500 \mathrm{MHz}, \mathrm{CDCl}_{3}\right): \delta 7.80(\mathrm{~s}, 1 \mathrm{H}), 7.67(\mathrm{~d}, J=6.5 \mathrm{~Hz}, 1 \mathrm{H}), 7.50$ $(\mathrm{d}, J=7.0 \mathrm{~Hz}, 1 \mathrm{H}), 7.38(\mathrm{t}, J=6.0 \mathrm{~Hz}, 1 \mathrm{H}), 6.09(\mathrm{br}, \mathrm{NH}, 2 \mathrm{H}) \cdot{ }^{13} \mathrm{C}$ NMR $\left(125 \mathrm{MHz}, \mathrm{CDCl}_{3}\right) \delta 167.9,135.0,134.8,132.0,129.9,127.7,125.3$.

4-Iodobenzamide (2p)<smiles>NC(=O)c1ccc(I)cc1</smiles> 
(Eluent: 40\% EtOAc/hexane); 95\% yield (469.6 mg); white solid; ${ }^{1} \mathrm{H}$ NMR $\left(600 \mathrm{MHz}, \mathrm{CDCl}_{3}\right): \delta 7.82(\mathrm{~d}, J=7.5 \mathrm{~Hz}, 2 \mathrm{H}), 7.54(\mathrm{~d}, J=7.5 \mathrm{~Hz}$, $2 \mathrm{H}), 6.02(\mathrm{br}, \mathrm{NH}, 2 \mathrm{H}) .{ }^{13} \mathrm{C}$ NMR $(150 \mathrm{MHz}$, DMSO-d 6 ) $\delta 167.7,137.6$, $132.0,129.9,118.5,99.4$.

[1, 1'-Biphenyl]-4-carboxamide (2q)<smiles>NC(=O)c1ccc(-c2ccccc2)cc1</smiles>

(Eluent: 40\% EtOAc/hexane); 77\% yield (304.9 mg); white solid; ${ }^{1} \mathrm{H}$ $\operatorname{NMR}\left(600 \mathrm{MHz}, \mathrm{CDCl}_{3}\right): \delta 7.90(\mathrm{~d}, J=7.0 \mathrm{~Hz}, 2 \mathrm{H}), 7.67(\mathrm{~d}, J=7.0 \mathrm{~Hz}$, $2 \mathrm{H}), 7.61(\mathrm{~d}, J=6.5 \mathrm{~Hz}, 2 \mathrm{H}), 7.47(\mathrm{t}, J=6.5 \mathrm{~Hz}, 2 \mathrm{H}), 7.40(\mathrm{t}, J=6.0 \mathrm{~Hz}$, $1 \mathrm{H}), 6.11$ (br, NH, 2H). ${ }^{13} \mathrm{C}$ NMR (150 MHz, DMSO-d $)$ ) $\delta 168.0,143.2$, $139.7,133.6,129.5,128.6,128.5,127.4,126.9$.

1-Naphthamide (2r)<smiles>NC(=O)c1cccc2ccccc12</smiles>

(Eluent: 40\% EtOAc/hexane); 69\% yield (235.9 mg); white solid; ${ }^{1} \mathrm{H}$ $\operatorname{NMR}\left(500 \mathrm{MHz}, \mathrm{CDCl}_{3}\right): \delta 8.44(\mathrm{~d}, J=7.0 \mathrm{~Hz}, 1 \mathrm{H}), 7.95(\mathrm{~d}, J=6.5 \mathrm{~Hz}$, $1 \mathrm{H}), 7.72(\mathrm{~d}, J=6.5 \mathrm{~Hz}, 1 \mathrm{H}), 7.59(\mathrm{t}, J=7.0 \mathrm{~Hz}, 1 \mathrm{H}), 7.47(\mathrm{t}, J=6.5$ $\mathrm{Hz}, 1 \mathrm{H}), 6.12$ (br, NH, 2H). ${ }^{13} \mathrm{C}$ NMR $\left(125 \mathrm{MHz}, \mathrm{CDCl}_{3}\right) \delta 171.5,133.7$, $133.0,131.2,130.0,128.3,127.3,126.5,125.4,124.6$.

Thiophene-2-carboxamide (3a)<smiles>NC(=O)c1cccs1</smiles>

(Eluent: 30\% EtOAc/hexane); 89\% yield (226.8 mg); white solid; ${ }^{1} \mathrm{H}$ $\operatorname{NMR}\left(600 \mathrm{MHz}, \mathrm{CDCl}_{3}\right): \delta 7.54(\mathrm{t}, J=5.0 \mathrm{~Hz}, 2 \mathrm{H}), 7.10(\mathrm{t}, J=3.5 \mathrm{~Hz}$, $1 \mathrm{H}), 5.80$ (br, $\mathrm{NH}, 2 \mathrm{H}) .{ }^{13} \mathrm{C} \mathrm{NMR}\left(150 \mathrm{MHz}, \mathrm{CDCl}_{3}\right) \delta 163.3,137.6$, $131.0,129.3,127.8$.

Thiophene-3-carboxamide (3b)<smiles>NC(=O)c1ccsc1</smiles>

(Eluent: 30\% EtOAc/hexane); 87\% yield (221.0 mg); white solid; ${ }^{1} \mathrm{H}$ NMR $\left(600 \mathrm{MHz}, \mathrm{CDCl}_{3}\right): \delta 7.91(\mathrm{~s}, 1 \mathrm{H}), 7.40(\mathrm{~d}, J=4.0 \mathrm{~Hz}, 1 \mathrm{H}), 7.36(\mathrm{t}$, $J=4.0 \mathrm{~Hz}, 1 \mathrm{H}), 5.84(\mathrm{br}, \mathrm{NH}, 2 \mathrm{H}) .{ }^{13} \mathrm{C}$ NMR $\left(150 \mathrm{MHz}, \mathrm{CDCl}_{3}\right) \delta 164.5$, $136.4,129.3,126.7,126.3$.

Picolinamide (3c)<smiles>NC(=O)c1cccnc1</smiles>

(Eluent: $45 \%$ EtOAc/hexane); $60 \%$ yield $(146.3 \mathrm{mg})$; white solid; ${ }^{1} \mathrm{H}$ NMR $\left(500 \mathrm{MHz}, \mathrm{CDCl}_{3}\right): \delta 8.58(\mathrm{~d}, J=4.5 \mathrm{~Hz}, 1 \mathrm{H}), 8.20(\mathrm{~d}, J=7.5 \mathrm{~Hz}$, $1 \mathrm{H}), 7.86(\mathrm{~m}, 1 \mathrm{H}), 7.45(\mathrm{~m}, 1 \mathrm{H}), 6.10(\mathrm{br}, \mathrm{NH}, 2 \mathrm{H}) .{ }^{13} \mathrm{C} \mathrm{NMR}(125 \mathrm{MHz}$, $\left.\mathrm{CDCl}_{3}\right) \delta 166.9,149.5,148.3,137.2,126.4,122.4$.

Nicotinamide (3d)<smiles>NC(=O)c1cccnc1</smiles>

(Eluent: 45\% EtOAc/hexane); $51 \%$ yield $(124.2 \mathrm{mg})$; white solid; ${ }^{1} \mathrm{H}$ NMR $\left(600 \mathrm{MHz}, \mathrm{CDCl}_{3}\right): \delta 9.03(\mathrm{~s}, 1 \mathrm{H}), 8.76(\mathrm{~d}, J=3.5$ $\mathrm{Hz}, 1 \mathrm{H}), 8.17(\mathrm{~d}, J=6.5 \mathrm{~Hz}, 1 \mathrm{H}), 7.42(\mathrm{t}, J=4.5 \mathrm{~Hz}, 1 \mathrm{H}), 6.28(\mathrm{br}$, $\mathrm{NH}, 2 \mathrm{H}) .{ }^{13} \mathrm{C}$ NMR $\left(150 \mathrm{MHz}, \mathrm{CDCl}_{3}\right) \delta 167.3,152.7,148.2,135.5$, $129.1,123.6$

Isonicotinamide (3e)<smiles>NC(=O)c1ccncc1</smiles>

(Eluent: $45 \%$ EtOAc/hexane); $65 \%$ yield (158.4 mg); white solid; ${ }^{1} \mathrm{H}$ NMR (600 MHz, $\left.\mathrm{CDCl}_{3}\right): \delta 8.78(\mathrm{~d}, J=4.5 \mathrm{~Hz}, 2 \mathrm{H}), 7.65(\mathrm{q}, J=2.5 \mathrm{~Hz}$, $2 \mathrm{H}), 6.18(\mathrm{br}, \mathrm{NH}, 2 \mathrm{H}) .{ }^{13} \mathrm{C} \mathrm{NMR}\left(150 \mathrm{MHz}, \mathrm{CDCl}_{3}\right) \delta 167.1,150.7$, 140.4, 121.0.

Pyrazine-2-carboxamide (3f)<smiles>NC(=O)c1cnccn1</smiles>

(Eluent: 50\% EtOAc/hexane); 68\% yield (167.3 mg); white solid; ${ }^{1} \mathrm{H}$ NMR (500 MHz, $\left.\mathrm{CDCl}_{3}\right): \delta 9.43(\mathrm{~s}, 1 \mathrm{H}), 8.78(\mathrm{~d}, J=2.5 \mathrm{~Hz}, 1 \mathrm{H}), 8.56$ $(\mathrm{d}, J=1.5 \mathrm{~Hz}, 1 \mathrm{H}), 5.98$ (br, NH, 2H). ${ }^{13} \mathrm{C}$ NMR $\left(125 \mathrm{MHz}, \mathrm{CDCl}_{3}\right) \delta$ 165.3, 147.5, 144.6, 144.1, 142.7 .

4-(Imidazo [1, 2-a] pyridin-2-yl) benzamide (3g)<smiles>NC(=O)c1ccc(-c2cn3ccccc3n2)cc1</smiles>

(Eluent: $70 \%$ EtOAc/hexane); $57 \%$ yield $(270.1 \mathrm{mg})$; white solid; ${ }^{1} \mathrm{H}$ NMR (600 MHz, DMSO-d d $_{6}: \delta 8.56(\mathrm{~d}, J=4.5 \mathrm{~Hz}, 1 \mathrm{H}), 8.52(\mathrm{~s}, 1 \mathrm{H})$, $8.04(\mathrm{t}, J=5.5 \mathrm{~Hz}, 3 \mathrm{H}), 7.97(\mathrm{~d}, J=5.5 \mathrm{~Hz}, 2 \mathrm{H}), 7.63(\mathrm{~d}, J=7.0 \mathrm{~Hz}, 1 \mathrm{H})$, $7.38(\mathrm{~s}, 1 \mathrm{H}), 7.31(\mathrm{t}, J=5.0 \mathrm{~Hz}, 1 \mathrm{H}), 6.95(\mathrm{~s}, 1 \mathrm{H}) .{ }^{13} \mathrm{C} \mathrm{NMR}(150 \mathrm{MHz}$, $\left.\mathrm{CDCl}_{3}\right) \delta 168.1,145.2,143.5,136.7,133.8,128.5,127.6,126.2,125.7$, 117.0, 113.2, 110.7.

Acetamide (3h)<smiles>CC(N)=O</smiles> 
(Eluent: $40 \%$ EtOAc/hexane); $58 \%$ yield $(68.9 \mathrm{mg})$; white solid; ${ }^{1} \mathrm{H}$ $\operatorname{NMR}\left(600 \mathrm{MHz}, \mathrm{CDCl}_{3}\right): 5.82(\mathrm{br}, \mathrm{NH}, 2 \mathrm{H}), 1.98(\mathrm{~s}, 3 \mathrm{H}) .{ }^{13} \mathrm{C} \mathrm{NMR}(125$ $\left.\mathrm{MHz}, \mathrm{CDCl}_{3}\right) \delta 172.9,22.7$.

Pentanamide (3i)<smiles>CCCCC(N)=O</smiles>

(Eluent: $40 \%$ EtOAc/hexane); 59\% yield (119.4 mg); white solid; ${ }^{1} \mathrm{H}$ NMR (600 MHz, DMSO- $\left.\mathrm{d}_{6}\right): \delta 7.23(\mathrm{br}, \mathrm{NH}, 2 \mathrm{H}) 2.02(\mathrm{t}, J=6.5 \mathrm{~Hz}$, $2 \mathrm{H}), 1.45(\mathrm{t}, J=6.0 \mathrm{~Hz}, 2 \mathrm{H}), 1.26(\mathrm{q}, J=6.0 \mathrm{~Hz}), 0.86(\mathrm{t}, J=5.5 \mathrm{~Hz}, 3 \mathrm{H})$. ${ }^{13} \mathrm{C}$ NMR (150 MHz, DMSO- $\left.\mathrm{d}_{6}\right) \delta 174.9,27.7,22.3,14.2$.

\section{Acknowledgment}

CSIR-CSMCRI communication No. 40/2018. Authors are thankful to "Analytical Discipline and Centralized Instrumental Facilities" for providing instrumentation facilities. We thank DST, Government of India. EMR/2016/000010) and CSIR-CSMCRI (OLP-0087/88/MLP0027) for financial support.

\section{References}

1. Bhattacharya A, Scott BP, Nasser N, Ao H, Maher MP, et al. (2007) Pharmacology and Antitussive Efficacy of 4-(3-Trifluoromethyl- pyridin-2-yl)-piperazine-1-carboxylic Acid (5-Trifluoromethyl- pyridin-2-yl)-amide (JNJ17203212), a Transient Receptor Potential Vanilloid 1 Antagonist in Guinea Pigs. J Pharmacol Exp Ther 323: 665-674. [Crossref]

2. Rivara S, Lodola A, Mor M, Bedini A, Spadoni G, et al. (2007) $N$-(Substitutedanilinoethyl)amides: Design, Synthesis, and Pharmacological Characterization of a New Class of Melatonin Receptor Ligands. J Med Chem 50: 6618-6626. [Crossref]

3. Deopura BL, Gupta B, Joshi M, Alagirusami R (2008) Polyesters and Polyamides; CRC Press: Boca Raton.

4. Das S, Wendt B, Moller K, Junge K, Beller M (2012) Two Iron Catalysts are Better than One: A General and Convenient Reduction of Aromatic and Aliphatic Primary Amides. Angew Chem Int Ed 51: 1662-1666. [Crossref]

5. Carey JS, Laffan D, Thomson C, Williams MT (2006) Analysis of the reactions used for the preparation of drug candidate molecules. Org Biomol Chem 4: 2337-2347. [Crossref]

6. Whitford D (2005) Proteins: Structure and Function Wiley, New York.

7. Sewald N, Jakubke HD (2009) Peptides: Chemistry and Biology Wiley, New York.

8. Pattabiraman VR, Bode JW (2011) Rethinking amide bond synthesis. Nature 480: 471479. [Crossref]

9. Zheng JS, Tang S, Huang YC, Liu L (2013) Development of New Thioester Equivalents for Protein Chemical Synthesis. Acc Chem Res 46: 2475-2584. [Crossref]

10. Lanigan RM, Starkov P, Sheppard TD (2013) Direct Synthesis of Amides from Carboxylic Acids and Amines Using B $\left(\mathrm{OCH}_{2} \mathrm{CF}_{3}\right)_{3}$. J Org Chem 78: 4512-4523. [Crossref]

11. Mabermann CE (1991) in Encyclopedia of Chemical Technology, Vol. 1 (Ed.: J. I. Kroschwitz), Wiley, New York, p. $251-266$.

12. Lipp D (1991) in Encyclopedia of Chemical Technology, Vol. 1 (Ed.:J. I. Kroschwitz), Wiley, New York, pp: 266-287.

13. Opsahl R (1991) in Encyclopedia of Chemical Technology, Vol. 2 (Ed.:J. I. Kroschwitz), Wiley, New York, pp: 346-356.

14. Dine TME, Erb W, Berhault Y, Rouden J, Blanchet J (2015) Catalytic Chemical Amide Synthesis at Room Temperature: One More Step Toward Peptide Synthesis. J Org Chem 80: 4532-4544. [Crossref]

15. Bannwart L, Abele S, Tortoioli S (2016) Metal-Free Amidation of Acids with Formamides and T3P. Synthesis 48: 2069-2078.

16. Gernigon N, Al-Zoubi, RM, Hall DG (2012) Direct Amidation of Carboxylic Acids Catalyzed by ortho-Iodo Arylboronic Acids: Catalyst Optimization, Scope, and Preliminary Mechanistic Study Supporting a Peculiar Halogen Acceleration Effect. $J$ Org Chem 77: 8386-8400.
17. de Figueiredo RM, Suppo JS, Campagne JM (2016) Nonclassical Routes for Amide Bond Formation. Chem Rev 116: 12029-12122. [Crossref]

18. McPherson CG, Caldwell N, Jamieson C, Simpsonb I, Watsona AJB (2017) Amidation of unactivated ester derivatives mediated by trifluoroethanol. Org Biomol Chem 15: 3507-3518. [Crossref]

19. Ekoue-Kovi K, Wolf C (2007) Metal-Free One-Pot Oxidative Amination of Aldehydes to Amides. Org Lett 9: 3429-3432. [Crossref]

20. Ghosh SC, Ngiam JSY, Seayad AM, Tuan DT, Johannes CW, et al. (2013) Tandem oxidative amidation of benzyl alcohols with amine hydrochloride salts catalysed by iron nitrate. Tetrahedron Lett 54: 4922-4925.

21. Tillack A, Rudloff I, Beller M (2001) Catalytic Amination of Aldehydes to Amides. Eur J Org Chem 523-528.

22. Achar TK, Mal P (2015) Radical-Induced Metal and Solvent-Free Cross-Coupling Using TBAI-TBHP: Oxidative Amidation of Aldehydes and Alcohols with $\mathrm{N}$-Chloramines via C-H Activation. J Org Chem 80: 666-672. [Crossref]

23. Fang $\mathrm{C}$, Li M, Hu X, Mo W, Hu B, et al. (2016) A Mild TEMPO-Catalyzed Aerobic Oxidative Conversion of Aldehydes into Nitriles. Adv Synth Catal 358: 1157-1163.

24. Liu H, Yan N, Dyson PJ (2014) Acid-free regio selective aminocarbonylation of alkenes. Chem Commun 50: 7848-7851. [Crossref]

25. Xu T, Sha F, Alper H (2016) Highly Ligand-Controlled Regioselective Pd-Catalyzed Aminocarbonylation of Styrenes with Aminophenols. J Am Chem Soc 138: 6629-6635. [Crossref]

26. Khamarui S, Maiti R, Maiti DK (2015) General base-tuned unorthodox synthesis of amides and ketoesters with water. Chem Commun 51: 384-387. [Crossref]

27. Battilocchio C, Hawkins JM, Ley SV (2014) Mild and Selective Heterogeneous Catalytic Hydration of Nitriles to Amides by Flowing through Manganese Dioxide. Org Lett 16: 1060-1063. [Crossref]

28. Marce P, Lynch J, Blacker AJ, Williams JMJ (2016) A mild hydration of nitriles catalysed by copper(II) acetateChem Commun 52: 1436-1438. [Crossref]

29. Zhang S, Xu H, Lou C, Senan AM, Chen ZG, et al. (2017) Efficient bimetallic catalysis of nitrile hydration to amides with a simple $\mathrm{Pd}(\mathrm{OAc})_{2} /$ lewis acid catalyst at ambient temperature. Eur J Org Chem 1870-1875.

30. Moorthy JN, Singhal N (2005) Facile and Highly Selective Conversion of Nitriles to Amides via Indirect Acid-Catalyzed Hydration Using TFA or $\mathrm{AcOH}-\mathrm{H}_{2} \mathrm{SO}_{4} . J \mathrm{Org}$ Chem 70: 1926-1929. [Crossref]

31. Deng T, Wang CZ (2017) Efficient Hydration of Nitriles Promoted by Gallic Acid Derived from Renewable Bioresources. ChemCatChem 9: 1349-1353.

32. Veisi H, Maleki B, Hameliana M, Ashrafib SS (2015) Chemoselective hydration of nitriles to amides using hydrated ionic liquid (IL) tetrabutylammonium hydroxide (TBAH) as a green catalyst. RSC Adv 5: 6365-6371.

33. Li Y, Chen H, Liu J, Wana X, Xu Q (2016) Clean synthesis of primary to tertiary carboxamides by $\mathrm{CsOH}$-catalyzed aminolysis of nitriles in water. Green Chem 18 : 4865-4870.

34. Midya GC, Kapat A, Maiti S, Dash J (2015) Transition-Metal-Free Hydration of Nitriles Using Potassium tert-Butoxide under Anhydrous Conditions. J Org Chem 80: 4148-4151.

35. Verma PK, Kumar N, Sharma U, Bala M, Kumar V, et al. (2013) Transition MetalFree Sodium Borohydride Promoted Controlled Hydration of Nitriles to Amides. Synth Commun 43: 2867-2875.

36. Chen H, Dai W, Chen Y, Xu Q, Chen J, et al. (2014) Efficient and selective nitrile hydration reactions in water catalyzed by an unexpected dimethylsulfinyl anion generated in situ from CsOH and DMSO. Green Chem 16: 2136-2141.

37. Tu T, Wang Z, Liu Z, Feng X, Wang Q (2012) Efficient and practical transition metalfree catalytic hydration of organo nitriles to amides. Green Chem 14: 921-924.

38. Sahnoun S, Messaoudi S, Peyrat JF, Brion JD, Alami M (2012) $\mathrm{Cs}_{2} \mathrm{CO}_{3}$ in pyrrolidinone promoted hydration of functionalized (hetero) aryl nitriles under metal-free conditions. Tetrahedron Lett 53: 2860-2863.

39. Niemeier JK, Rothhaar RR, Vicenzi JT, Werner JA (2014) Application of Kinetic Modeling and Competitive Solvent Hydrolysis in the Development of a Highly Selective Hydrolysis of a Nitrile to an Amide Org Process Res Dev 18: 410.

40. Rao SN, Mohan DC, Adimurthy S (2013) L-Proline: An Efficient Catalyst for Transamidation of Carboxamides with Amines. Org Lett 15: 1496-1499. [Crossref] 
41. Rao SN, Mohan DC, Adimurthy S (2014) Chitosan: an efficient recyclable catalyst for transamidation of carboxamides with amines under neat conditions. Green Chem 16: 4122-4126.

42. Rao SN, Mohan DC, Adimurthy S (2016) AIBN-promoted amidation of anilines with 1 , 3-diketones via oxidative cleavage of $\mathrm{C}-\mathrm{C}$ bond under aerobic conditions. Tetrahedron 72: 4889-4894.

43. Pappula V, Ravi C, Samanta S, Adimurthy S (2017) Oxidative Amidation of Methylarenes and Heteroamines under Metal-Free Conditions. ChemistrySelect 2: 5887-5890.

44. Rao SN, Reddy NNK, Samanta S, Adimurthy S (2017) I Catalyzed Oxidative Amidation of Benzylamines and Benzyl Cyanides under Mild Conditions. J Org Chem 82: 13632-13642. [Crossref]
45. Li X, Li B, You J, Lan J (2013) Copper-catalysed oxidative C-H/N-H cross-coupling between formamides and amides through chelation-assisted $\mathrm{N}-\mathrm{H}$ activation. Org Biomol Chem 11: 1925-1928.

46. Bolhofer WA, Deana AA, Habecker CN, Hoffman JM Gould NP, et al. (1983) Inhibitors of gastric acid secretion: antisecretory 2-pyridylurea derivatives. J Med Chem 26: 538544. [Crossref]

47. Patel OPS, Anand D, Maurya RK, Yadav PP (2015) Copper-catalyzed highly efficient oxidative amidation of aldehydes with 2 -aminopyridines in an aqueous micellar system. Green Chem 17: 3728-3732.

48. Ragupathi A, Sagadevan A, Lin CC, Hwu JR, Hwang KC (2016) Copper (I) - catalysed oxidative $\mathrm{C}-\mathrm{N}$ coupling of 2 -aminopyridine with terminal alkynes featuring a $\mathrm{C}$ triple bond $\mathrm{C}$ bond cleavage promoted by visible light. Chem Commun 52: 11756-11759.

Copyright: (C2018 Naresh KRN. This is an open-access article distributed under the terms of the Creative Commons Attribution License, which permits unrestricted use, distribution, and reproduction in any medium, provided the original author and source are credited. 